\title{
Optimización del proceso de recuperación de metales valiosos presentes en los residuos de la amalgama dental
}

\section{Process Optimization for Valuable Metal Recovery from Dental Amalgam Residues}

\author{
C.M. Parra-Mesa \\ Grupo Ciencia y Tecnología Biomédica (CTB) \\ Universidad de Antioquia. Medellín, Colombia. \\ E-mail:cmparra@udea.edu.co \\ J. Ruiz-Cordoba \\ Grupo Ciencia y Tecnología Biomédica (CTB) \\ Universidad de Antioquia. Medellín, Colombia. \\ E-mail:jruiz@udea.edu.co \\ J.I. Pérez-Rave \\ Grupo Ciencia y Tecnología Biomédica (CTB) \\ Universidad de Antioquia. Medellín, Colombia. \\ E-mail:Ejipr056@udea.edu.co \\ M.E. Carmona-Arango \\ Grupo Ciencia y Tecnología Biomédica (CTB) \\ Universidad de Antioquia. Medellín, Colombia. \\ E-mail:rosendatory@gmail.com \\ G.J. Gómez-Mejía \\ Empresa New Stetic S.A. Medellin, Colombia. \\ E-mail:gigomez@newstetic.com \\ R. Carmona-Correa \\ Empresa New Stetic S.A. Medellín, Colombia. \\ E-mail:rcarmona@newstetic.com \\ J.A. Muñoz-Tabarez \\ Empresa New Stetic S.A. Medellín, Colombia. \\ E-mail:jruiz@udea.edu.co
}

(Recibido: mayo de 2007; aceptado: abril de 2008)

\section{Resumen}

En este artículo se exhibe la metodología empleada en la optimización del proceso de lixiviación en una planta semipiloto para la recuperación de los metales valiosos presentes en los residuos de la amalgama dental. En la primera etapa se usó el diseño factorial con el propósito de caracterizar el proceso, y en la segunda, el diseño central compuesto rotacional de segundo orden para modelar el porcentaje de cobre disuelto en función de la concentración de ácido nítrico, el tiempo de lixiviación y la temperatura. El modelo explicó el $81 \%$ de la variabilidad en la respuesta, porcentaje que se considera satisfactorio dada la complejidad de la cinética del proceso y, porque además, permitió definir las condiciones de operación para la mayor recuperación 
DOI: http://dx.doi.org/10.22201/fi.25940732e.2009.10n3.020

Optimización del proceso de recuperación de metales valiosos presentes en los residuos de la amalgama dental

del cobre, la cual experimentalmente fue del $99.15 \%$, lograda a temperatura de $55^{\circ} \mathrm{C}$, concentración de $30 \%$ en peso y tiempo de 26 horas.

Descriptores: Diseño experimental, diseño factorial, amalgama dental, lixiviación, optimización.

\begin{abstract}
In this paper, the methodology used for optimizing leaching in a semi pilot plant is presented. This leaching process was applied to recover value metals from dental amalgam residues. $2^{3}$ factorial design was used to characterize the process during the first stage and in the second one, a central compound rotational design was used for modeling copper percentage dissolved, a function of the nitric acid concentration, leaching time and temperature. This model explained the $81 \%$ of the response variability, which is considered satisfactory given the complexity of the process kinetics and, furthermore, it allowed the definition of the operation conditions for better copper recovery, which this was of $99.15 \%$, at a temperature of $55^{\circ} \mathrm{C}$, a concentration of $30 \%$ by weight and a time of 26 hours.
\end{abstract}

Keywords: Experimental design, factorial design, dental amalgam, leaching, optimization.

\section{Introducción}

La recuperación y posterior reciclaje de los residuos, sean peligrosos o no, es la mejor forma de velar por la protección de los recursos naturales y el ambiente; además, se logra en la mayoría de los casos la reinserción a la cadena productiva de los materiales recuperados.

La amalgama dental es una aleación compuesta por mercurio líquido (42-52\%), plata (20-36\%), cobre (1-15\%), estaño (13-16\%) y en ocasiones también contiene el zinc en mínimo porcentaje (Lorscheider et al., 1995). Debido principalmente al contenido de mercurio, los residuos de la amalgama dental generados en los consultorios odontológicos, producto de la eliminación de restauraciones usadas o sobrantes de restauraciones nuevas, origina un problema ambiental importante, $y$ desde luego, se constituye en un factor de alto riesgo para la salud de las personas laboralmente expuestas (Halbach et al., 2000).

La empresa New Stetic S.A., fabricante de aleaciones para amalgama dental, entre otros productos odontológicos, desarrolló un proyecto en conjunto con la Universidad de Antioquia (Colombia), para sensibilizar y concientizar a la comunidad odontológica del departamento de Antioquia en lo concerniente al manejo de la amalgama y a la disposición final de los residuos de la misma, provenientes de los consultorios odontológicos. Fruto de esta alianza nació el proyecto de diseñar y construir una planta semipiloto para el tratamiento de los mencionados residuos, mediante la destilación del mercurio y la lixiviación con ácido nítrico de la aleación resultante. Además, un propósito básico de la investigación y que se constituye en el objeto del presente artículo, fue la optimización del proceso de recuperación de los metales valiosos presentes en los residuos de la amalgama. En dicho desarrollo, participaron por parte de la Universidad, los grupos de investigación Ciencia y Tecnología Biomédica (CTB) y de Investigaciones Pirometalúrgicas y de Materiales (GIPIMME).

\section{Metodología}

La metodología se desarrolló en tres etapas fundamentales:

\section{Elección de los factores y región inicial de experimentación}

En un proceso intervienen gran número de factores y, de acuerdo con el principio de Pareto (Domínguez, 1995), tan solo una minoría tiene incidencia significativa en la efectividad del mismo. Para comprender de una mejor manera la cinética del proceso, se adelantó una revisión bibliográfica con el propósito de identificar los posibles factores de mayor criticidad, establecer la región inicial de experimentación y, así mismo, definir la variable a través de la cual mediría la efectividad del sistema.

Como resultado de dicha etapa, se encontró que los factores de mayor criticidad son: la temperatura, concentración de ácido nítrico y el tiempo de disolución (Evered, 1971; Xammel et al., 1981), obviándose la 
DOI: http://dx.doi.org/10.22201/fi.25940732e.2009.10n3.020

C.M. Parra-Mesa, J. Ruiz-Cordoba, J.I. Pérez-Rave, M.E. Carmona-Arango, G.J. Gómez-Mejía, R. Carmona-Correa y J.A. Muñoz-Tabarez

agitación, debido a que sólo influye en la lixiviación a muy bajas velocidades, además, luego de exceder un determinado valor $(400 \mathrm{rpm})$ se vuelve independiente de la disolución (Palacios, 2002).

Por otro lado, se halló que la recuperación porcentual de cobre es la que determina la efectividad del proceso, por lo que su cinética de disolución es más lenta que la de los demás metales (Muñoz, 2004), y en consecuencia, determina la rapidez de reacción. Igualmente en esta fase, mediante revisión literaria y experiencia del equipo investigador en el tema, se identificaron los niveles de los factores a estudiar y se presentan en el siguiente apartado.

\section{Caracterización del proceso:}

El propósito de esta fase fue identificar mediante criterios estadísticos, cuáles de los factores seleccionados en la etapa anterior, afectan significativamente la variable respuesta: \% disuelto de cobre y, de la misma manera, obtener información pertinente sobre la dirección de operación de dichos factores, que posibilite acercar la región experimental hacia condiciones óptimas de funcionamiento.

Para tal efecto, se empleó el diseño factorial $2^{\mathrm{K}}$, donde $\mathrm{K}$, representa el número de factores de interés, cada uno con dos niveles de resultado (alto, bajo). En la tabla 1 se presentan las bases del diseño y la región inicial de experimentación.

Tabla 1. Bases del diseño y región inicial de experimentación

\begin{tabular}{|c|c|c|c|}
\hline \multicolumn{3}{|c|}{ Número de factores experimentales } & 3 \\
\hline \multicolumn{3}{|c|}{ Número de variables respuesta } & 1 \\
\hline \multicolumn{3}{|c|}{ Número de corridas } & 8 \\
\hline \multirow{2}{*}{ Factores } & \multicolumn{2}{|c|}{ Nivel } & \multirow{2}{*}{ Unidades } \\
\hline & Bajo & Alto & \\
\hline Temperatura & 26 & 50 & ${ }^{\circ} \mathrm{C}$ \\
\hline Concentración $\mathrm{HNO}_{3}$ & 33.3 & 50 & $\%$ \\
\hline Tiempo & 18 & 28 & Horas \\
\hline \multicolumn{4}{|l|}{ Variable respuesta: } \\
\hline \multicolumn{3}{|c|}{ Cobre disuelto } & $\%$ \\
\hline
\end{tabular}

Para concluir sobre los efectos de los tres factores de interés y sus interacciones, se empleó la prueba de análisis de varianza, la cual particiona la variabilidad de los datos en sus principales componentes: una atribuida al error dentro de los tratamientos y la otra a la variabilidad entre los tratamientos (Montgomery, 1991).

$$
\text { Optimización del proceso }
$$

Para determinar las condiciones óptimas de operación de los factores potenciales del diseño, que condujera a la maximización de la proporción de cobre disuelto, se empleó la técnica de superficie de respuesta, puesto que posibilita el análisis y modelado de sistemas donde la finalidad es optimizar una variable respuesta influenciada por diversos factores.

En el caso de tres factores significativos, $X_{1}, X_{2}, X_{3}$, la superficie de respuesta $Y$ está representada por la forma funcional:

$$
E(Y)=f\left(X_{1}, X_{2}, X_{3}\right)
$$

El ajuste de la superficie mencionada se llevó a cabo mediante un diseño central compuesto rotacional de segundo orden, cuyas condiciones experimentales vienen dadas por las de un diseño factorial $2^{k}$, -empleado en la fase anterior-, aumentando $2 \mathrm{~K}$ puntos axiales, y uno más en el centro del espacio experimental. En este último se realizaron 6 réplicas para estimar la varianza y evaluar la relevancia de los factores de interés (Ruíz et al., 2003)

Este diseño, es mucho más potente que el empleado en la fase de caracterización, ya que permite incluir en el modelo términos cuadráticos y superar una posible falta de ajuste obtenida en el diseño $2^{k}$. La relación funcional para aproximar el modelo a la mencionada superficie se exhibe en la ecuación 2.

$$
E(Y)=\beta_{0}+\sum_{i=1}^{k} \beta_{i} X_{i}+\sum_{i=1}^{k} \beta_{i i} X_{i}^{2}+\sum_{i=1}^{k} \sum_{j=1}^{L} \beta_{i j} X_{i} X_{j}
$$

donde $\beta_{i}$ y $\beta_{i i}$, corresponden respectivamente a los efectos lineal y cuadrático del factor $X_{i} \mathrm{y}, \beta_{i j}$ informa sobre el efecto de la interacción entre los factores $X_{i}$ y $X_{j}$.

El ajuste de la superficie de interés y la estimación de los parámetros del modelo se llevó a cabo mediante el software estadístico Statgraphics 5.1

\section{Resultados y análisis}

La presentación de los resultados y análisis seguirá el orden de las fases experimentales: caracterización del proceso y optimización. 


\section{Caracterización del proceso}

En la tabla 2 se muestran los resultados experimentales para los 8 ensayos correspondientes al diseño factorial $2^{3}$, cuyo orden de ejecución fue establecido de manera aleatoria.

Cabe resaltar que la mayor proporción de cobre disuelto $(96,54 \%)$ se obtuvo en el ensayo 3 , cuyas condiciones de temperatura, tiempo y concentración fueron $50{ }^{\circ} \mathrm{C}$ (alto), 28 horas (alto) y 33\% (bajo), respectivamente. Del mismo modo, el resultado menos satisfactorio $(54,04 \%)$ se dio a una temperatura de $26^{\circ} \mathrm{C}$, un tiempo de 18 horas y una concentración de 50\%.

En la figura 1, se ilustran las combinaciones de los niveles de los tres factores de interés y la recuperación de cobre en cada combinación.

Figura 1. Representación cúbica de la recuperación de cobre

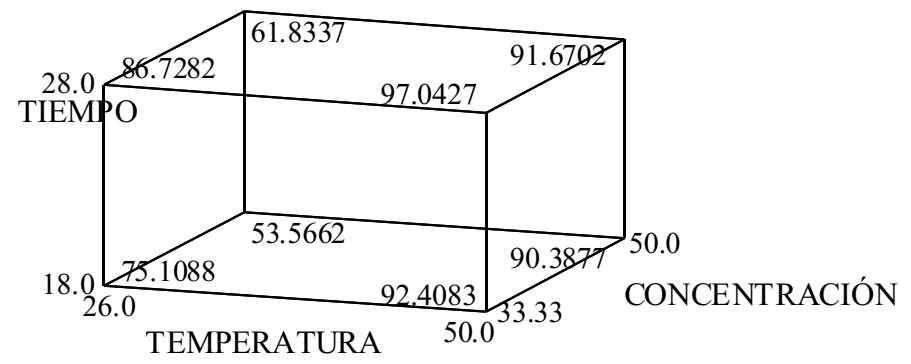

Tabla 2. Condiciones de los ensayos y resultados experimentales de la etapa de caracterización

\begin{tabular}{ccccc}
\hline Ensayo Num. & Temperatura $\left({ }^{\circ} \mathrm{C}\right)$ & Tiempo (horas) & Concentración de HNO3 $(\%)$ & \% de Cu total disuelto \\
\hline 1 & 50 & 28 & 50 & 92.15 \\
2 & 50 & 18 & 50 & 89.92 \\
3 & 50 & 28 & 33 & 96.54 \\
4 & 50 & 18 & 33 & 92.87 \\
5 & 26 & 28 & 50 & 61.33 \\
6 & 26 & 18 & 50 & 54.04 \\
7 & 26 & 28 & 33 & 87.21 \\
8 & 26 & 18 & 33 & 74.62 \\
\hline
\end{tabular}

Tabla 3. Estimación de los efectos

\begin{tabular}{clc}
\hline Factor & & Efecto \\
\hline Promedio & $=$ & 81.0932 \\
A: Temperatura & $=$ & 23.568 \\
B: Concentración & $=$ & -13.4575 \\
C: Tiempo & $=$ & 6.451 \\
AB & $=$ & 9.761 \\
AC & $=$ & -3.4925 \\
BC & $=$ & -1.676 \\
ABC & 0.9595 \\
\hline
\end{tabular}


$\mathrm{Al}$ analizar la estimación de los efectos de los factores potenciales del diseño y de sus interacciones sobre la recuperación del cobre (tabla 3), se puede deducir la mayor importancia que tienen: la temperatura, la concentración y la interacción entre ellas, y en menor medida, el tiempo y sus interacciones con los otros dos factores. A modo de ejemplarizar la interpretación, el efecto de la temperatura significa que, al variar dicho factor de su nivel mínimo $\left(26^{\circ} \mathrm{C}\right)$ a su nivel máximo $\left(50^{\circ} \mathrm{C}\right)$, el promedio de recuperación de cobre se incrementa en un $23.568 \%$.

De la tabla 3 también se observa que interacciones $\mathrm{ABC}, \mathrm{AC}$ y $\mathrm{BC}$ tienen un efecto mínimo, por lo cual, con el propósito de determinar la significancia estadística de los efectos de los factores mediante el análisis de varianza, se asumió que la variabilidad atribuida a tales interacciones es de naturaleza aleatoria. Como consecuencia de lo anterior, y de acuerdo con el principio de los efectos esparcidos (Montgomery, 2004), se combinaron los grados de libertad y la suma de cuadrados de las anteriores fuentes para dar origen a una estimación de la varianza del error experimental. La tabla 4 contiene los resultados del análisis de varianza siguiendo el procedimiento antes descrito.
Como puede observarse de la columna valor-P, los factores: temperatura, concentración y su interacción $A B$, tienen valores-P inferiores a 0.05 , reflejando que tienen influencia significativa en la recuperación del cobre. De la misma manera, el tiempo, a pesar de presentar un valor-P levemente superior al valor crítico, se prefirió mantenerlo como factor potencial del diseño, puesto que está presente en el proceso incidiendo de manera importante en los costos del mismo y, además, la región estudiada en esta primera fase corresponde a una región de exploración.

En la figura 2, se ilustra la interacción entre la temperatura y la concentración de $\mathrm{HNO}_{3}$, y muestra que las mayores recuperaciones de cobre se pueden obtener con concentraciones menores de 33,33 y temperaturas superiores a $50^{\circ} \mathrm{C}$, lo cual se constituyó en la base para definir la nueva región de experimentación respecto a estos dos factores. Respecto al tiempo, prefrió mantenerse el rango de experimentación.

Tabla 4. Análisis de varianza para la recuperación de cobre en el diseño factorial $2^{3}$

\begin{tabular}{|c|c|c|c|c|c|}
\hline Factor & Suma cuadrados & g.1 & Madia cuadrados & $\mathrm{F}$ & Valor-p \\
\hline A: Temperatura & 1110.9 & 1 & 1110.9 & 104.62 & 0.0020 \\
\hline B: Concentración & 362.209 & 1 & 362.209 & 3411 & 0.0100 \\
\hline C: Tiempo & 83.2308 & 1 & 83.2308 & 7.84 & 0.0679 \\
\hline $\mathrm{AB}$ & 190.554 & 1 & 190.554 & 17.95 & 0.0241 \\
\hline Total error & 31.8543 & 3 & 10.6181 & & \\
\hline Total (corr.) & 1778.75 & 7 & & & \\
\hline
\end{tabular}

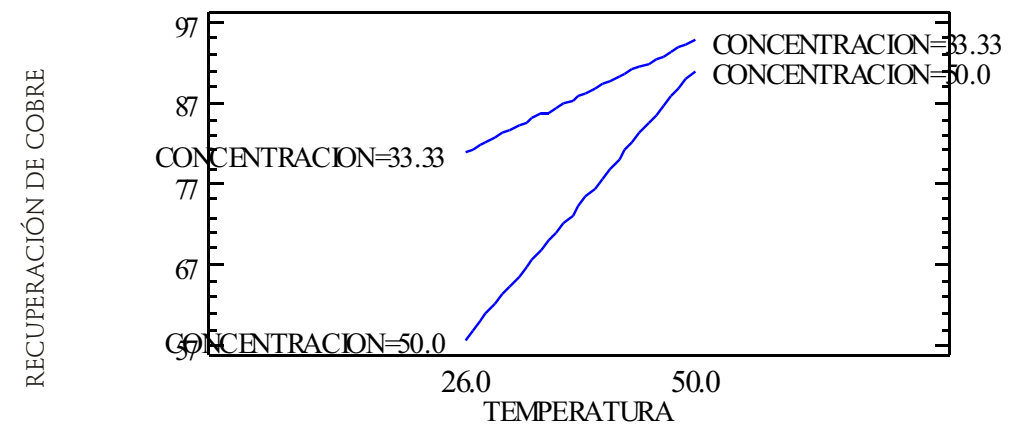

Figura 2. Interacción entre la temperatura y la concentración 
DOI: http://dx.doi.org/10.22201/fi.25940732e.2009.10n3.020

Optimización del proceso de recuperación de metales valiosos presentes en los residuos de la amalgama dental

\section{Optimización del proceso}

Con base en la información obtenida en la etapa de caracterización se redefinió la región de operación de los factores potenciales del diseño (tabla 5), con el fin de optimizar el proceso.
En la tabla 6 se presentan las diferentes combinaciones de los niveles de los factores, así como los resultados obtenidos en la nueva región de experimentación.

A partir de la tabla 6, se puede observar que la mayor recuperación porcentual de cobre (99.15\%) se logró bajo una temperatura de $55^{\circ} \mathrm{C}$, una concentración de ácido

Tabla 5. Bases del diseño empleado en la optimización

Nombre del diseño: Central compuesto rotacional de segundo orden

Número de factores experimentales: 3

Número de variables respuesta: 1

Número de corridas: 20

Grados de libertad del error: 10

\begin{tabular}{lcccc}
\hline \multicolumn{1}{c}{ Factores } & Nivel inferior & Niveles axiales & Nivel central & Nivel superior \\
\hline Temperatura $\left({ }^{\circ} \mathrm{C}\right)$ & 45 & 41.6 y 58.4 & 50 & 55 \\
Concentración & 18 & 13.9 y 34.1 & 24 & 30 \\
HNO3 (\%) & 18 & 15.3 y 28.7 & 22 & 26 \\
Tiempo (horas) & &
\end{tabular}

Tabla 6. Condiciones de los ensayos y resultados experimentales de la etapa de optimización (continúa...)

\begin{tabular}{ccccc}
\hline Ensayo Num. & Temperatura $\left({ }^{\circ} \mathrm{C}\right)$ & Concentración $(\%)$ & Tiempo (horas) & Cu Total disuelto (\%) \\
\hline 1 & 55 & 18 & 18 & 95.04 \\
2 & 45 & 30 & 18 & 91.00 \\
3 & 50 & 24 & 28.72 & 97.56 \\
4 & 50 & 24 & 22 & 93.26 \\
5 & 45 & 18 & 26 & 91.22 \\
6 & 45 & 30 & 26 & 89.61 \\
7 & 50 & 24 & 22 & 91.97 \\
8 & 50 & 13.92 & 22 & 93.78 \\
9 & 55 & 30 & 18 & 93.24 \\
10 & 50 & 34.08 & 22 & 94.05 \\
11 & 55 & 30 & 26 & 99.15 \\
12 & 45 & 18 & 18 & 82.03 \\
13 & 50 & 24 & 22 & 94.52 \\
14 & 50 & 24 & 22 & 95.33 \\
15 & 58.41 & 24 & 22 & 97.92 \\
16 & 50 & 24 & 22 & 94.88 \\
17 & 50 & 24 & 22 & 93.95 \\
\hline
\end{tabular}


DOI: http://dx.doi.org/10.22201/fi.25940732e.2009.10n3.020

C.M. Parra-Mesa, J. Ruiz-Cordoba, J.I. Pérez-Rave, M.E. Carmona-Arango, G.J. Gómez-Mejía, R. Carmona-Correa y J.A. Muñoz-Tabarez

Tabla 6. Condiciones de los ensayos y resultados experimentales de la etapa de optimización (... continuación)

\begin{tabular}{ccccc}
\hline Ensayo Num. & Temperatura $\left({ }^{\circ} \mathrm{C}\right)$ & Concentración $(\%)$ & Tiempo (horas) & Cu Total disuelto $(\%)$ \\
\hline 18 & 50 & 24 & 15.28 & 91.95 \\
19 & 55 & 18 & 26 & 94.81 \\
20 & 41.59 & 24 & 22 & 85.53 \\
\hline
\end{tabular}

nítrico de 30\% y un tiempo de 26 horas, y la menor recuperación fue de $82.03 \%$. En otras palabras, el rango de variación en esta nueva etapa (17,12\%), fue mucho menor que el arrojado en la etapa de caracterización (42.5\%). Por último, cabe resaltar que el $85 \%$ de los resultados experimentales presentan una recuperación porcentual de cobre superior al $90 \%$, lo que indica que efectivamente la región de experimentación trabajada cobija las condiciones óptimas de funcionamiento del proceso.

$\mathrm{Al}$ ajustar la superficie de respuesta descrita por la ecuación 2, los resultados del análisis de varianza mostrados en la tabla 7, señalan que la concentración en esta región no tiene influencia significativa, como sí la tienen el tiempo y la temperatura, existiendo además en esta última, un efecto cuadrático.

De la misma manera que en la etapa de caracterización, se procedió a reunir las fuentes de variación no significativas con la del error, para dar lugar a una mejor estimación de la varianza del término error aleatorio. Los resultados de tal procedimiento se muestran en la tabla 8.

Tabla 7. Análisis de varianza para la recuperación de cobre en la etapa de optimización

\begin{tabular}{lccccc}
\hline \multicolumn{1}{c}{ Fuente } & Suma de cuadrados & gl & Cuadrado medio & F-ratio & Valor-P \\
\hline A: temperatura & 177.373 & 1 & 177.373 & 35.74 & 0.0001 \\
B: Concentración & 7.85003 & 1 & 7.85003 & 1.58 & 0.2371 \\
C: Tiempo & 38.4489 & 1 & 38.4489 & 7.75 & 0.0193 \\
AA & 19.6277 & 1 & 19.6277 & 3.95 & 0.0748 \\
AB & 2.90405 & 1 & 2.90405 & 0.59 & 0.4620 \\
AC & 0.5618 & 1 & 0.5618 & 0.11 & 0.7435 \\
BB & 2.22303 & 1 & 2.22303 & 0.45 & 0.5185 \\
BC & 2.4642 & 1 & 2.4642 & 0.50 & 0.4971 \\
CC & 0.132179 & 1 & 0.132179 & 0.03 & 0.8736 \\
\hline Total error & 49.6311 & 10 & 4.96311 & & \\
\hline Total (corr.) & 300.006 & 19 & & \\
\hline
\end{tabular}

Tabla 8. Análisis de varianza final para la recuperación de cobre (continúa...)

\begin{tabular}{lccccc}
\hline \multicolumn{1}{c}{ Fuente } & Suma de cuadrados & gl & Cuadrado medio & F-Ratio & Valor-P \\
\hline A. Temperatura & 177.373 & 1 & $177-373$ & 46.01 & 0.0000 \\
B: Concentración & 7.85003 & 1 & 7.85003 & 2.04 & 0.1741 \\
C: Tiempo & 38.4489 & 1 & 38.4489 & 9.97 & 0.0065 \\
AA & 18.5034 & 1 & 18.5034 & 4.80 & 0.0447 \\
Total error & 57.8311 & 15 & 3.85541 & & \\
\hline
\end{tabular}


DOI: http://dx.doi.org/10.22201/fi.25940732e.2009.10n3.020

Optimización del proceso de recuperación de metales valiosos presentes en los residuos de la amalgama dental

Tabla 8. Análisis de varianza final para la recuperación de cobre (... continuación)

\begin{tabular}{ccccc}
\hline Fuente & Suma de cuadrados & gl & Cuadrado medio & F-Ratio \\
\hline Total (corr.) & 300.006 & 19 & & \\
\hline
\end{tabular}

R-squared $=80.7234 \%$

R-squared (adjusted for d.f.) $=75.5829$

Gráfico de paretto para la recuperación de cobre

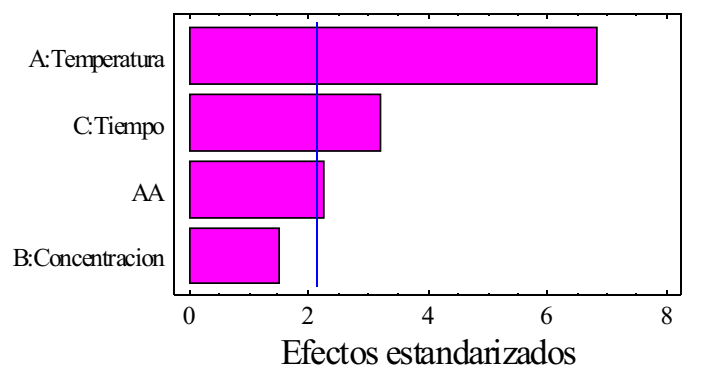

Figura 3. Gráfico de Paretto

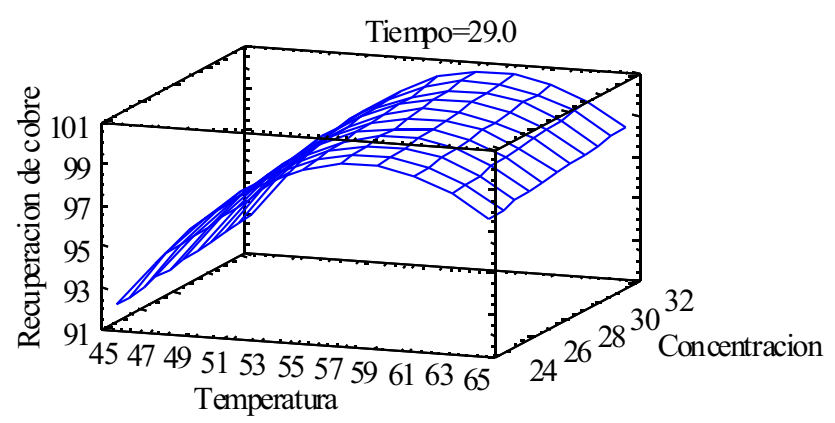

Figura 4. Gráfico de superficie de respuesta para la recuperación de cobre

La importancia de los diversos factores considerados finalmente, puede también deducirse del gráfico de $\mathrm{Pa}$ retto que se presenta en la figura 3 , donde los factores e interacciones que más pesan presentan barras con alturas que superan la línea vertical.

La ecuación de regresión que estima el promedio de recuperación de cobre, de acuerdo a los factores seleccionados está representada en la ecuación 3 , siendo la predicción del porcentaje de recuperación de cobre.

$$
\hat{Y}=-66.72+5.21 A+0.13 B+0.42 C-0.05 A^{2} .
$$

De la tabla 8 , se puede observar que tal modelo explica aproximadamente el $81 \%$ de la variabilidad en las respuestas del porcentaje de cobre recuperado.
Empleando procedimientos matemáticos sobre la superficie ajustada (ecuación 3) se concluyó que la optimización de la recuperación porcentual de cobre (teóricamente del $100 \%$ ) se da en cercanías de la condición: temperatura de $58{ }^{\circ} \mathrm{C}$, concentración de $31 \%$ y tiempo de 29 horas, tal como puede verse del gráfico de superficie de respuesta (figura 4); sin embargo, una conclusión definitiva sobre la condición óptima puede obtenerse experimentando en una zona más refinada, alrededor de la mencionada condición.

Dicho procedimiento no se efectuó puesto que la organización empresarial quedó totalmente satisfecha con la condición experimental que permitió obtener un $99.15 \%$ de recuperación de cobre. 
DOI: http://dx.doi.org/10.22201/fi.25940732e.2009.10n3.020

C.M. Parra-Mesa, J. Ruiz-Cordoba, J.I. Pérez-Rave, M.E. Carmona-Arango, G.J. Gómez-Mejía, R. Carmona-Correa y J.A. Muñoz-Tabarez

\section{Discusión}

El mercurio es hoy un metal que por sus efectos nocivos, incluso a nivel genético, es motivo de especial atención por los estados y organismos ambientales que buscan todos los días mayores controles en el uso de dicho elemento. En la presente investigación subyace un resultado que merece resaltarse, y es precisamente el beneficio que recibe la sociedad, cuando se integra la academia y el sector productivo para aportar soluciones expertas a problemas que afectan la vida humana, animal y vegetal, como es el caso del tratamiento inadecuado de los residuos de la amalgama dental.

La amalgama dental se viene utilizando hace más de 150 años y, todavía hoy, no se le ha encontrado un sustituto que presente las propiedades mecánicas que la hacen ventajosa, a pesar que existen varios materiales restauradores alternativos que están siendo objeto de amplia investigación y con un uso que va en aumento. Por ello, la mejor forma de manejar los residuos de la misma es mediante la recuperación del mercurio y demás metales valiosos que la conforman, de modo que puedan reinsertarse a la cadena productiva, generando riqueza. La experimentación hace parte del proceso de conocimiento, y cuando ella es diseñada para producir resultados objetivos y pertinentes que puedan analizarse empleando métodos estadísticos, se constituye en una herramienta eficiente y eficaz para aprender acerca del fenómeno que se estudia.

El trabajo del diseño e implementación de la planta semipiloto como resultado de este proyecto, no sólo da respuesta a la necesidad sentida en Colombia de dar tratamiento y disposición final adecuados a los residuos de amalgama dental, sino que también en el cumplimiento de las regulaciones ambientales vigentes.

\section{Conclusiones}

Empleando dos diseños experimentales, se logró encontrar una condición donde la recuperación del cobre presente en los residuos de la amalgama dental fue de $99.15 \%$. Así mismo, una región que debe incluir el punto donde se dé el 100\%, en razón del modelo estadístico que permitió explicar satisfactoriamente los resultados de la recuperación por los valores en los que se fijaron los factores temperatura, concentración de ácido nítrico y tiempo de lixiviación. Dicho resultado, no solo contribuye a la minimización del impacto negativo al ambiente, sino que también genera beneficios importantes para la empresa usuaria.

\section{Referencias}

Lorscheider F., Vimy M.J., Summers A.O., Zwiers H. The Dental Amalgam Mercury Controversy -Inorganic Mercury and the CNS; Linkage of Mercury and Antibiotic Resistance in Intestinal Bacteria. Toxicology, 97:19-22. 1995.

Halbach S., Welzl G., Kremers L., Willruth H., Mehl A., Wack F.X. et al. Steady-State Transfer and Depletion Kinetics of Mercury from Amalgam Fillings. Sci Total Environ, 259:13-21. 2000.

Domínguez J. Dirección de operaciones, aspectos tácticos y operativos en la producción y los servicios. España. McGraw Hill. 1995. P. 424.

Evered, S. The Treatment of Gold Silver Bearing Materials from Mines and Other Sources. In: Bulletin South African Institute of Assayers and Analyst. January. Pp. 4-15. 1971.

Palacios C. Lixiviación de menas auríferas con sales oxidantes en medio acido mediante el proceso severo [en línea] 2002. Disponible en: http://sisbib.unmsm.edu.pe/bibvirtualdata/publicaciones/geologia/vol5_n9_2002/lixiviacion_menas.pdf

Muñoz, Alejandro. Diseño e implementación de una planta semipiloto para el tratamiento de residuos de amalgama dental. Universidad de Antioquia, Medellín-Colombia. 2004.

Montgomery D. Diseño y análisis de experimentos. México. Grupo Editorial Iberoamérica. 1991. P. 50.

Ruíz et al. Modelamiento del proceso de recubrimiento de instrumental quirúrgico con cromoduro. En: Revista Facultad de Ingeniería. Universidad de Antioquia. Colombia. N³0. Diciembre. P 90. 2003.

Montgomery D. Diseño y análisis de experimentos. México. Segunda edición. Editorial LIMUSA SA. 2004. P. 245.

Xammel R., Hans-Wilhelm L. Electrolytic Recovery of Precious Metals from Dilute Solutions. En: Journal of Metals. Estados Unidos. Pp. 45-48. 1981. 
DOI: http://dx.doi.org/10.22201/fi.25940732e.2009.10n3.020

Optimización del proceso de recuperación de metales valiosos presentes en los residuos de la amalgama dental

\section{Semblanza de los autores}

Carlos Mario Parra-Mesa. Es estadístico MSc. Profesor investigador en la Universidad de Antioquia. Jefe del Departamento de Ingeniería Industrial y director del Grupo Ciencia y Tecnología Biomédica CTB.

Jairo Ruiz-Cordoba. Es ingeniero metalúrgico MSc. Asimismo, es profesor-investigador en la Universidad de Antoquia, coordinador del grupo de Materiales Precisos (MAPRE) de la Universidad de Antioquia y presidente de la Sociedad de Ingenieros Metalúrgicos y de Materiales de la Universidad de Antioquia (SIMEDUA).

Jorge Iván Pérez-Rave. Es ingeniero industrial, profesor-investigador Universidad de Antioquia. Codirector del Grupo Gestión de la Calidad y Miembro de Ciencia y Tecnología Biomédica CTB. Consultor en mejoramiento continuo.

María Eugenia Carmona-Arango. Es ingeniera metalúrgica y catedrática en la Universidad Pontificia Bolivariana. Es investigadora del Grupo de Materiales Precisos (MAPRE) de la Universidad de Antioquia e integrante de la Junta directiva de la Sociedad de Ingenieros Metalúrgicos y de Materiales de la Universidad de Antioquia (SIMDEUA).

Gabriel Jaime Gómez-Mejia. Es ingeniero metalúrgico. Labora en el área de investigación y desarrollo de la empresa privada New Stetic S.A., Antioquia Colombia.

Rosaura Carmona-Correa. Es ingeniera química. Labora en el área de investigación y desarrollo de la empresa privada New Stetic S.A., Antioquia Colombia.

José Alejandro Muñoz-Tabarez. Es ingeniero de materiales. Actualmente es aspirante a doctor en ingeniería de materiales. 